\title{
Introduction to cerebral cavernous malformation: a brief review
}

\author{
Jaehong $\operatorname{Kim}^{1,2, *}$ \\ ${ }^{1}$ Department of Biochemistry, School of Medicine, Gachon University, Incheon 21936, ${ }^{2}$ Department of Health Sciences and Technology, \\ Gachon Advanced Institute for Health Science and Technology, Gachon University, Incheon 21999, Korea
}

\begin{abstract}
The disease known as cerebral cavernous malformations mostly occurs in the central nervous system, and their typical histological presentations are multiple lumen formation and vascular leakage at the brain capillary level, resulting in disruption of the blood-brain barrier. These abnormalities result in severe neurological symptoms such as seizures, focal neurological deficits and hemorrhagic strokes. CCM research has identified 'Ioss of function' mutations of three ccm genes responsible for the disease and also complex regulation of multiple signaling pathways including the WNT/ $\beta$-catenin pathway, TGF- $\beta$ and Notch signaling by the $\mathrm{ccm}$ genes. Although CCM research is a relatively new and small scientific field, as CCM research has the potential to regulate systemic blood vessel permeability and angiogenesis including that of the blood-brain barrier, this field is growing rapidly. In this review, I will provide a brief overview of CCM pathogenesis and function of $\mathrm{ccm}$ genes based on recent progress in CCM research. [BMB Reports 2016; 49(5): 255-262]
\end{abstract}

\section{INTRODUCTION}

The vascular malformations characterizing the disease known as cerebral cavernous malformations (CCMs; OMIM \#116860, 603284, 603285) mostly occur in the central nervous system (CNS) and their typical histological presentations are single or multiple lumen formation and vascular leakage at the brain capillary level, aka disruption of the blood- brain barrier (BBB) (1). These abnormalities result in severe neurological symptoms such as hemorrhagic stroke (30-40\%), seizures (40-70\%), headache (10-30\%) and focal neurological symptoms (35-50\%) (2). Together with arteriovenous malformation (AVM), CCM is a major cerebral vascular disease entity, albeit showing milder phenotypes than AVM (around $50-80 \%$ of CCM cases are asymptomatic) $(3,4)$. Prevalence of both sporadic and familial

*Corresponding author. Tel: +82-32-899-6588; Fax: +82-32-8996039; E-mail: geretics@gachon.ac.kr

http://dx.doi.org/10.5483/BMBRep.2016.49.5.036

Received 19 February 2016

Keywords: Angiogenesis, Central nervous system, Cerebral cavernous malformation, Signaling, Vascular permeability type CCMs is estimated to be $0.1-0.5 \%$ in the general population and the proportion of familial cases in total CCM cases has been estimated to be as high as 50\% in Hispanic-American patients and close to $10-40 \%$ in other populations $(5,6)$.

So far, CCM research has been a small scientific field. However, as CCM research has a good potential to regulate systemic blood vessel permeability and angiogenesis (7-9), importantly those of the BBB and possibly tumor vasculature, the field is now rapidly growing (Fig. 1). Indeed, both in vivo and in vitro studies revealed that perturbation of the WNT/ $\beta$-catenin pathway $(10,11)$, TGF- $\beta / B M P(10,12,13)$ and Notch signaling (14), cytoskeletal regulation $(8,15)$ and anti-oxidant signaling (16-18) are responsible for CCM pathogenesis and several proteomic studies elegantly showed that all three $\mathrm{ccm}$ genes encode CCM proteins comprising distinct macromolecular complexes, implying complex regulation of multiple signaling pathways due to various interactions with many signaling molecules by each CCM protein (19-21). As individual proteins comprising the distinct macromolecular CCM complexes are still not fully characterized, our understanding of the composition of the CCM macromolecular complexes and associated functional networks is still in its infancy. The important unresolved questions in this field are as follows: 1) Why are the phenotypes almost exclusively seen in the CNS, although all the three ccm genes are ubiquitously expressed? 2) How do ccm genes act in formation and maintenance of neurovascular units? 3) What are the functions of $\mathrm{ccm}$ genes in non-endothelial cells and extra-CNS endothelial cells? and 4) How to identify the genetic or environmental modifiers that will address incomplete clinical penetrance of CCMs?

\section{MUTATIONS OF CCM GENES}

ccm1, ccm2 and ccm3 genes were identified in 1999 (22), 2003 (23) and 2005 (24), respectively. The three genes: ccm1 (Krit1; Krev interaction trapped 1), ccm2 (MGC4607, Malcavernin) and ccm3 (PDCD10), respectively, which are located on chromosomes 7q21.2, 7p13 and 3q25.2-q27 $(25,26)$, are known to be responsible for familial cases of CCMs and for more than half of the sporadic cases of CCM with multiple lesions $(27,28)$. Relative frequency of mutations of $\mathrm{ccm}$ genes in familial cases is about $53-65 \%, 15-19 \%$ and $10-22 \%$ for $\mathrm{ccm} 1$, ccm2 and ccm3, respectively (29-31) and familial CCM is an 
50

40

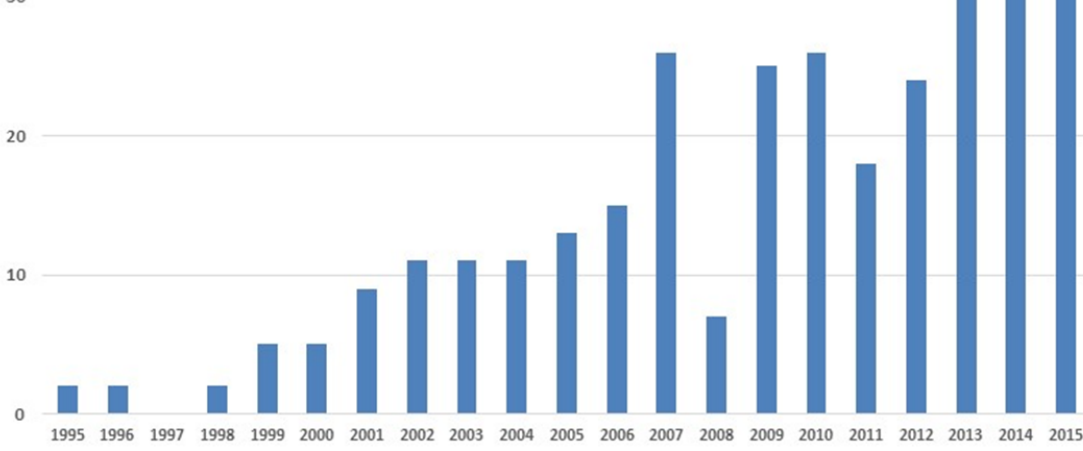

Fig. 1. Annual publication records of CCM from 1995 to 2015. PubMed search using keywords 'Krit1 or $\mathrm{ccm} 1$ or $\mathrm{ccm} 2$ or $\mathrm{ccm} 3$ or cerebral cavernous malformation' yielded 440 publications. autosomal dominant disease with incomplete clinical and radiological penetrance $(1,3,32)$. The existence of additional CCM loci has been suggested as $5-15 \%$ of familial cases cannot be explained by the three known ccm genes $(31,33)$. ccm mutations are also found in sporadic cases $(33,34)$ and sporadic cases with a single lesion, and not multiple CCM lesions appear to harbor far less ccm mutations $(35,36)$. Of note, the phenotypes of CCM3 patients or animal models are more severe than those of CCM1 or CCM2 patients or animal models (37-39).

So far, more than 100 distinct CCM1 mutations, 30 CCM2 mutations and $20 \mathrm{CCM} 3$ mutations have been identified and most of the $\mathrm{ccm}$ mutations lead to either a premature termination codon or large deletions, strongly suggesting that most of the ccm mutations are 'loss of function' mutations $(2,28)$.

\section{MECHANISMS OF CCM PATHOGENESIS}

It is becoming important to understand how CCM1, CCM2 and CCM3 function, what roles they play in signal transduction, and where do their signaling pathways overlap. The strong interaction between CCM1 and CCM2 appears to be important for the regulation of CCM signaling $(40,41)$ and evidences imply that the two CCM proteins participate in common signaling pathways (38). CCM3 appears to act in different signaling pathways $(37-39,42)$. Pathogenesis of CCM follows the Knudsonian two-hit mechanism, in which loss of one allele due to a germline mutation of one of the three known CCM genes in an affected cell (first hit) is accompanied with somatic mutation in the other (second hit) $(27,43-46)$. Increased vascular permeability was observed both in haplo-insufficient CCM1(+/-) and CCM2 (+/-) mouse endothelial cells in vitro and in lung and liver tissues of $\mathrm{CCM} 1+/-)$ and $\mathrm{CCM} 2$ $(+/-)$ animals in vivo (8), indicating the asymptomatic extra-CNS manifestations. Because only about $30 \%$ of humans with CCM lesions will eventually develop clinical symptoms, the existence of a modifier is suggested for the incomplete clinical penetrance.

Activation of RhoA and its effector, Rho kinase (ROCK), induces stress fiber formation, resultant decreased stability of adherens junction and abnormal extracellular matrix (ECM) remodeling, and increases endothelial permeability (47). Ablation of $\mathrm{ccm} 1, \mathrm{ccm} 2$ or $\mathrm{ccm} 3$ in endothelial cells has been shown to increase Rho activation (48). CCM1 and CCM2 loss resulted in destabilization of another CCM1 interacting protein, integrin cytoplasmic domain-associated protein-1 (ICAP-1), which increased $\beta 1$ integrin activation and led to increased RhoA-dependent contractility $(49,50)$ and commonly activated p38, Akt, and ERK $1 / 2$ in endothelial cells (42). Ras-related protein (Rap1)-dependent association of CCM1 with vascular endothelial cadherin at adherens junctions (AJs), with CCM1 dependent cortical cytoskeletal remodeling leads to EC barrier enhancement $(7,8,50)$. Aberrant Rho activation was also found in sporadic CCM patients $(8,43)$. In the early stage of CCM research, the reversal of Rho activation due to inhibition of ROCK in ccm-ablated endothelial cells suggested that Rho ac- 
tivation is a major mechanism in CCM pathogenesis (48). However, further studies revealed that Rho/ROCK signaling is not a unique target for CCM disease.

Recent studies elegantly demonstrated endothelial-mesenchymal transition (EndMT) in endothelial cells lining CCMs in tamoxifen-inducible CCM1 loss of function mice $(12,13)$. EndMT has been previously implicated in cardiac fibrosis and cancer progression and it leads to a modification of the endothelial cell phenotype, resulting in a loss of cellular junctions, acquisition of migratory properties, loss of endothelial-specific markers, and gain of mesenchymal markers. EndMT may occur as a result of upregulation of endogenous bone morphogenetic protein 6 (BMP6) and activation of the transforming growth factor (TGF)- $\beta$ and bone morphogenetic protein (BMP) signaling pathways. CCM1 is also a Notch activator (14) and loss of CCM1, ICAP1 and CCM3 has been shown to cause downregulation of Notch signaling, leading to increased angiogenesis (51-53). In line with these findings, overexpression of CCM1 caused Notch activation and decreased sprouting angiogenesis after stimulation with VEGF (51). Studies have shown that loss of CCM1-mediated Notch inhibition and Kruppel-like factor 4 (KLF4) induction result in upregulation of BMP6 and resultant EndMT $(13,51)$. Autophagy appears to be another important mechanism of CCM pathogenesis because ablation of ccm1, ccm 2 and ccm3 commonly causes mTOR-ULK1 pathway mediated suppression of autophagy and resultant EndMT (54). Ablation of $\mathrm{ccm} 1$ causes increased nuclear $\beta$-catenin localization and WNT signaling $(15,48)$ and Wnt-independent stimulation of $\beta$-catenin transcriptional activity precedes TGF/BMP signaling for EndMT (10). Another study revealed that an increase in nuclear $\beta$-catenin and VEGF signaling is observed when $\mathrm{ccm} 1$ and $\mathrm{ccm} 3$, but not $\mathrm{ccm} 2$, are ablated (53, 55). Involvement of CCM proteins in VEGF and Notch signaling suggests that the paracrine effect modulated by CCM may also affect non-endothelial cells in the lesion. Indeed, recent reports suggested that $\mathrm{ccm} 3$ ablation induced VEGF secretion activated Erk $1 / 2$ and AKT in endothelial cells $(42,56)$, and in a GBM xenograft mouse model, endothelial ccm3 ablation increased tumor progression due to increased proliferation of GBM cells, which indicate autonomous and non-autonomous roles of CCM proteins in tumor progression (56). Also, another report, which suggested that $\mathrm{ccm} 1$ knockdown in endothelial cells deregulated Notch signaling in adjacent pericytes, supports the notion (14).

Combinational effects and genetic modifiers may explain radiological and clinical incomplete penetrance of CCM. Combinational effects due to reduced expression or disturbed function of other proteins in CCM signaling have been shown in zebra fish (57), and it has been suggested that genetic susceptibility is related to oxidative stress $(3,58)$. CCM1 has been shown to modulate the expression level of the antioxidant protein SOD2, indicating a potential contribution of the oxidant pathway to CCM pathogenesis $(18,59)$. A recent report showed that inducible knockout of ccm2 gene after vessel de- velopment did not develop CCM lesions in a mouse model, and this suggests that the time window for genetic changes and also possibly, resultant specific changes in microvascular environment may be essential for the CCM phenotypes (60).

Various animal model systems including zebra fish, drosophila and mouse models are available for CCM studies $(8,38,60-65)$. In brief, CCM1(+/-)Msh2(-/-) (61) and CCM1 (+/-)p53(-/-) (66) mice were used to prove the Knudsonian two-hit mechanism. The CCM3 $(+/-)$ mouse model showed different pathogenetic mechanisms underlying CCM lesion genesis and echoing differences in severity between CCM1 or CCM2 and CCM3 disease $(42,67)$. Most significant phenotypes are observed due to ccm3 mutation (30). Many animal studies have been performed to identify the cellular component of the BBB; endothelial cells, neuroglial cells and smooth muscle cells, which is responsible for CCM pathogenesis. Inducible knockout experiments of ccm1, ccm2 and $\mathrm{ccm} 3$ genes showed that perturbed homeostasis of endothelial cells appears to be the most important for CCM phenotypes, albeit mice with Emx1-Cre, Gfap-Cre and Nestin-Cre induced neuronal cell specific knockout of ccm3 showed considerable CCM phenotypes.

\section{FORMATION OF A HETEROTRIMERIC CCM1-CCM2-CCM3 'CCM COMPLEX SIGNALING PLATFORM $^{\prime}$}

CCM proteins directly interact with each another to form a CCM1-CCM2-CCM3 based signaling platform $(68,69)$ with interacting proteins rather distinct for each CCM protein (19-21, 70-72). Interaction between CCM2 and CCM3 is necessary for stability of the two proteins (73) and CCM2 dependent stabilization of CCM1 has also been reported (49). CCM2 appears to act as the central hub in the formation of CCM complex by using its PTB domain and a conserved motif C-terminal of the PTB domain to interact with the $2^{\text {nd }}$ and $3^{\text {rd }} \mathrm{NPxY} / \mathrm{F}$ motifs of CCM1 and the focal adhesion targeting homology (FAT-H) domain of CCM3, respectively (74). A meticulous phosphomapping study has revealed that CCM2 has fourteen Ser/Thr phosphorylation sites with three sites on its PTB domain, suggesting that phosphorylation events may potentially influence formation of the CCM signaling complex (Fig. 2) (21). Although these three CCM proteins together can form the CCM signaling platform, the function of CCM3 appears to be somewhat different from that of the other CCM proteins. Proteomic studies showed that the interaction of CCM3 with members of the GCKIII family is more frequently detected than that of CCM3 with $\mathrm{CCM} 2(39,75)$. In summary, the interaction between CCM1 and CCM2 appears to be intrinsic to CCM complex function; however, the role of $\mathrm{CCM} 3$ in the CCM complex remains to be determined. Precise identification of proteins that interact with all three CCM proteins or either of the three CCM proteins is necessary to acquire a better understanding of the CCM complex signaling platforms.

CCM1 is a 736 amino acid protein, which was originally de- 


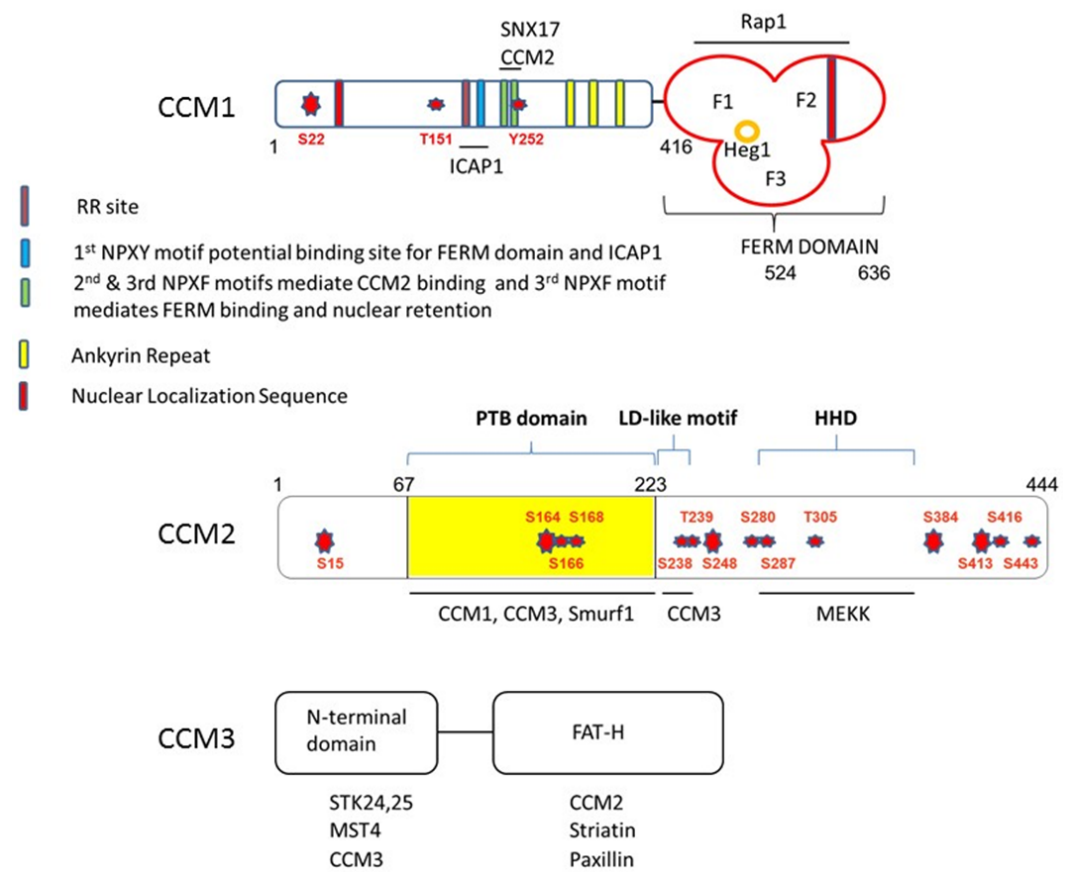

Fig. 2. Representative protein interaction and phosphorylation sites in CCM proteins. Number indicates the location of specific amino acid residue from $\mathrm{N}$-terminus. Size of red stars indicates relative abundance of phosphorylation on serine $(\mathrm{S})$, threonine $(\mathrm{T})$ or tyrosine (Y) residues. Based on (18). scribed to contain a c-terminal FERM (band 4.1, ezrin, radixin, moesin) domain that interacts with the small GTPase Krev-1 (Rap1) and Nd1-L (59), three PTB binding NPxY/F motifs and an ankyrin repeat domain (ARD) N-terminal to the FERM domain consisting of 4 ankyrin repeats. NPxY/F motifs $\left({ }^{192} \mathrm{NPAY}\right.$, ${ }^{231} \mathrm{NPLF},{ }^{250} \mathrm{NPYF}$ ) are important for the protein-protein interactions including intermolecular interactions with CCM2, Heg1 and ICAP1, and intramolecular CCM1 conformational changes and resultant functional outputs (75). CCM1 interaction with microtubules determines subcellular localization of CCM1 in the cytoplasm (76-78). After release from microtubules, CCM1 seems to localize to cell membranes driven predominantly by interaction with Rap1 $(78,79)$. The FERM domain of CCM1 is comprised of F1, F2 and F3 lobes. F1 and F2 lobes interact with Switch I and II regions of Rap1, and F1 and F3 lobes interact with the c-terminal cytoplasmic region of Heg1 $(80,81) .{ }^{231}$ NPLF and ${ }^{250}$ NPYF sequences, the second and third of the three CCM1 NPX (Y/F) motifs, are known to interact with the PTB domain of CCM2, and a recent study showed that the third motif is crucial for the interaction with a single binding site on the CCM2 PTB domain (41). The 2nd NPX (Y/F) motif in CCM1 interacts with the FERM domain of SNX17 (82)

CCM2, a 444 amino acid protein, has a N-terminal PTB domain, LD-like motif and C-terminal harmonin homology domain (HHD) (73). The $\alpha$-helical LD like motif within CCM2 binds the highly conserved HP1 pocket of the CCM3 FAT-H domain (73). CCM2 interacts with MEKK3 (64), CCM1 and CCM3 (69), and CCM2 either mediates the activation of
MEKK3 signaling in response to osmotic stress or negatively regulates MEKK3 signaling. Depletion of CCM2 phosphorylates MEKK3 and ERK5 and activates the transcriptional program downstream of MEKK3 (64). The CCM2-MEKK3 interaction is also known to be partially responsible for Rho-ROCK signaling (83). The PTB domain of CCM2 interacts with Smurf1, a ubiquitin ligase (E3), and the CCM2-Smurf1 interaction was shown to localize Smurf1 for degradation of RhoA (84).

The best identified role of CCM3 would be as a bridging factor within the striatin-interacting phosphatase and kinase (STRIPAK) complex that is essential for cell polarity and migration (73). A recent report, which showed that the CCM2-CCM3 interaction is required for endothelial cell network formation and that CCM3 in the absence of CCM2 is sufficient for endothelial cell growth, indicates a complex function of CCM3, both dependent and independent of CCM2 (73). CCM3 is a 212 amino acid protein with an $\mathrm{N}$-terminal dimerization domain and a C-terminal FAT-H domain (Fig. 2). A flexible hinge region links CCM3's N-terminal dimerization and C-terminal FAT-H domains. The FAT-H domain contains an exquisitely conserved hydrophobic patch 1 (HP1) site, and this site is important for interacting with LD-like motif of CCM2, the striatins (75) and paxillin (85). CCM3 can either homodimerize (86) or directly heterodimerize with each of the three GCKIII serine/threonine kinases: STK24 (MST3), STK25 (Ysk1; Sok1) and MST4 (MASK) (86-89). It has been suggested that the interaction of CCM3 with GCKIII kinases and with striatin, a regulatory subunit of the PP2A phosphatase holoenzyme, may cause CCM3 to act as a hub within the STRIPAK complex, 
bringing the GCKIII kinases to the STRIPAK phosphatase for the regulation of cell polarity, further linking CCM3 with vascular development $(70,90)$. CCM3 is localized to the cell membrane upon VEGF stimulation where it protects VEGFR2 from endocytosis (91), and CCM3 interaction with Phosphatidylinositol $(3,4,5)$-trisphosphate may play a role in CCM3 localization to the plasma membrane (92).

\section{CCM THERAPEUTICS}

Currently, there is no approved medical therapy for treating CCM other than surgical resection (3). Readers are advised to refer to a recent review that provides detailed information about CCM management including diagnosis and surgical and conservative treatment (93). Recent studies including whole genome sequencing studies have suggested that both sporadic cases with multiple lesions and familial cases of CCM have a common genetic underpinning of the two-hit mutation mechanism in the ccm genes and that the majority, if not all, of these sporadic cases with multiple lesions are really genetic cases (43). These findings imply that both familial and part of sporadic cases of CCM may be amenable to the same medical therapy.

Chemical inhibition of Rho activity in endothelial cells rescued CCM phenotypes in vitro (48) and administration of fasudil, a Rho-kinase inhibitor, resulted in fewer, smaller, and less hemorrhagic lesions in mice with CCM1 mutations. This was the first report of successful pharmacologic therapy in a CCM animal model (8) and the results were reproduced in a separate report (94). Statin therapy was suggested for CCM and it showed symptomatic improvement in a mouse model (50). Inhibition of HMG-CoA reductase by statin not only decreases cholesterol production, but also reduces geranyl-geranyl-pyrophosphate (GGPP), necessary for the isoprenylation of RhoA, critical for tethering RhoA to the cell membrane and activation of the small GTPase. However, statin administration was associated with an increased risk of intracerebral hemorrhage (95) and CCM patients receiving statin medications for routine cardiovascular indications showed lower permeability in brain white matter, but not in lesion (96). These findings indicate that the clinical application of statin does not appear to be feasible at this moment. It is worthwhile to mention about the inhibitors of TGF signaling. In the CCM1 mouse model, LY-364947, an inhibitor of TGF- $\beta$ type I receptors and phosphorylation of SMAD signaling, significantly reduced phosphorylated SMAD1 levels and inhibited the EndMT switch (12). The combination of this inhibitor and SB-431542 (another inhibitor of SMAD phosphorylation) reduced the number of vascular malformations and prevented vascular "leakage". Sulindac, a FDA approved, non-steroidal and anti-inflammatory drug, can control the development of CCM lesions in CCM3 knockout mice through suppression of beta-catenin activity (10).

\section{CONCLUSIONS AND PERSPECTIVE}

Because functional manipulation of CCM signaling has a good potential for regulating systemic blood vessel permeability and angiogenesis (7-9), importantly that of the BBB and possibly tumor vasculature, the research field of CCM is now growing rapidly. However, our understanding of the composition of the CCM macromolecular complexes and associated functional networks is still in its infancy. Also, we have no understanding of the phenotypes that may arise from increased expression of CCM proteins. In our laboratory, we observed increased expression of $\mathrm{ccm}$ genes during the progression of prostate cancer, potentially implicating the involvement of ccm genes in cancer signaling(data not shown). I expect that further studies will reveal how the formation of the CCM signaling platform is regulated and also provide answers to important unresolved questions in this field.

\section{ACKNOWLEDGEMENTS}

I sincerely apologize for not being able to cite the work of colleagues in many other important related studies because of space restrictions. This study was supported by the Basic Science Research Program through the National Research Foundation of Korea (NRF) funded by the Ministry of Science, ICT \& Future Planning (NRF-2014R1A1A1004682) and by the Gachon University research fund of 2015 (GCU2015-5103).

\section{REFERENCES}

1. Cavalcanti DD, Kalani MY, Martirosyan NL, Eales J, Spetzler RF and Preul MC (2012) Cerebral cavernous malformations: from genes to proteins to disease. J Neurosurg $116,122-132$

2. Kumar A, Bhandari A and Goswami C (2014) Surveying genetic variants and molecular phylogeny of cerebral cavernous malformation gene, CCM3/PDCD10. Biochem Biophys Res Commun 455, 98-106

3. Choquet H, Pawlikowska L, Lawton MT and Kim H (2015) Genetics of cerebral cavernous malformations: current status and future prospects. J Neurosurg Sci 59, 211-220

4. Labauge $P$, Denier $C$, Bergametti $F$ and Tournier-Lasserve E (2007) Genetics of cavernous angiomas. Lancet Neurol $6,237-244$

5. Rigamonti D, Hadley MN, Drayer BP et al (1988) Cerebral cavernous malformations. Incidence and familial occurrence. N Engl J Med 319, 343-347

6. Pozzati E, Acciarri N, Tognetti F, Marliani F and Giangaspero F (1996) Growth, subsequent bleeding, and de novo appearance of cerebral cavernous angiomas. Neurosurgery 38, 662-670

7. Meliton A, Meng F, Tian Y, Shah AA, Birukova AA and Birukov KG (2015) Role of Krev Interaction Trapped-1 in Prostacyclin-Induced Protection against Lung Vascular Permeability Induced by Excessive Mechanical Forces and Thrombin Receptor Activating Peptide 6. Am J Respir Cell 
Mol Biol 53, 834-843

8. Stockton RA, Shenkar R, Awad IA and Ginsberg MH (2010) Cerebral cavernous malformations proteins inhibit Rho kinase to stabilize vascular integrity. J Exp Med 207, 881-896

9. Stamatovic SM, Sladojevic N, Keep RF and Andjelkovic AV (2015) PDCD10 (CCM3) regulates brain endothelial barrier integrity in cerebral cavernous malformation type 3: role of CCM3-ERK1/2-cortactin cross-talk. Acta Neuropathol 130, 731-750

10. Bravi L, Rudini N, Cuttano R et al (2015) Sulindac metabolites decrease cerebrovascular malformations in CCM3-knockout mice. Proc Natl Acad Sci U S A 112, 8421-8426

11. Glading AJ and Ginsberg MH (2010) Rap1 and its effector KRIT1/CCM1 regulate beta-catenin signaling. Dis Model Mech 3, 73-83

12. Maddaluno L, Rudini N, Cuttano R et al (2013) EndMT contributes to the onset and progression of cerebral cavernous malformations. Nature 498, 492-496

13. Cuttano R, Rudini N, Bravi L et al (2015) KLF4 is a key determinant in the development and progression of cerebral cavernous malformations. EMBO Mol Med 8, 6-24

14. Schulz GB, Wieland E, Wustehube-Lausch J et al (2015) Cerebral Cavernous Malformation-1 Protein Controls DLL4-Notch3 Signaling Between the Endothelium and Pericytes. Stroke 46, 1337-1343

15. Glading A, Han J, Stockton RA and Ginsberg MH (2007) KRIT-1/CCM1 is a Rap1 effector that regulates endothelial cell cell junctions. J Cell Biol 179, 247-254

16. Moglia A, Goitre L, Gianoglio S et al (2015) Evaluation of the bioactive properties of avenanthramide analogs produced in recombinant yeast. Biofactors 41, 15-27

17. Goitre L, De Luca E, Braggion S et al (2014) KRIT1 loss of function causes a ROS-dependent upregulation of c-Jun. Free Radic Biol Med 68, 134-147

18. Goitre L, Balzac F, Degani S et al (2010) KRIT1 regulates the homeostasis of intracellular reactive oxygen species. PLoS One 5, e11786

19. Baxter SS, Dibble CF, Byrd WC et al (2014) Role of cytoskeletal proteins in cerebral cavernous malformation signaling pathways: a proteomic analysis. Mol Biosyst 10, 1881-1889

20. Jung KH, Han DM, Jeong SG, Choi MR, Chai YG and Cho GW (2015) Proteomic analysis reveals KRIT1 as a modulator for the antioxidant effects of valproic acid in human bone-marrow mesenchymal stromal cells. Drug Chem Toxicol 38, 286-292

21. Kim J, Sherman NE, Fox JW and Ginsberg MH (2011) Phosphorylation sites in the cerebral cavernous malformations complex. J Cell Sci 124, 3929-3932

22. Sahoo T, Johnson EW, Thomas JW et al (1999) Mutations in the gene encoding KRIT1, a Krev-1/rap1a binding protein, cause cerebral cavernous malformations (CCM1). Hum Mol Genet 8, 2325-2333

23. Liquori CL, Berg MJ, Siegel AM et al (2003) Mutations in a gene encoding a novel protein containing a phosphotyrosine-binding domain cause type 2 cerebral cavernous malformations. Am J Hum Genet 73, 1459-1464

24. Bergametti F, Denier C, Labauge P et al (2005) Mutations within the programmed cell death 10 gene cause cerebral cavernous malformations. Am J Hum Genet 76, 42-51

25. Craig HD, Gunel M, Cepeda O et al (1998) Multilocus linkage identifies two new loci for a mendelian form of stroke, cerebral cavernous malformation, at 7p15-13 and 3q25.2-27. Hum Mol Genet 7, 1851-1858

26. Dubovsky J, Zabramski JM, Kurth J et al (1995) A gene responsible for cavernous malformations of the brain maps to chromosome 7q. Hum Mol Genet 4, 453-458

27. Riant F, Bergametti $F$, Ayrignac X, Boulday G and Tournier-Lasserve E (2010) Recent insights into cerebral cavernous malformations: the molecular genetics of CCM. FEBS J 277, 1070-1075

28. Draheim KM, Fisher OS, Boggon TJ and Calderwood DA (2014) Cerebral cavernous malformation proteins at a glance. J Cell Sci 127, 701-707

29. Spiegler S, Najm J, Liu J et al (2014) High mutation detection rates in cerebral cavernous malformation upon stringent inclusion criteria: one-third of probands are minors. Mol Genet Genomic Med 2, 176-185

30. Denier C, Labauge P, Bergametti F et al (2006) Genotypephenotype correlations in cerebral cavernous malformations patients. Ann Neurol 60, 550-556

31. Riant F, Cecillon M, Saugier-Veber $P$ and TournierLasserve E (2013) CCM molecular screening in a diagnosis context: novel unclassified variants leading to abnormal splicing and importance of large deletions. Neurogenetics 14, 133-141

32. Mondejar R and Lucas M (2015) Molecular diagnosis in cerebral cavernous malformations. Neurologia [Epub ahead of print]

33. D'Angelo R, Marini V, Rinaldi C et al (2011) Mutation analysis of CCM1, CCM2 and CCM3 genes in a cohort of Italian patients with cerebral cavernous malformation. Brain Pathol 21, 215-224

34. D'Angelo R, Alafaci C, Scimone C et al (2013) Sporadic cerebral cavernous malformations: report of further mutations of CCM genes in 40 Italian patients. Biomed Res Int 2013, 459253

35. Tsutsumi S, Ogino I, Miyajima M et al (2013) Genomic causes of multiple cerebral cavernous malformations in a Japanese population. J Clin Neurosci 20, 667-669

36. Verlaan DJ, Laurent SB, Sure U et al (2004) CCM1 mutation screen of sporadic cases with cerebral cavernous malformations. Neurology 62, 1213-1215

37. Chan AC, Drakos SG, Ruiz OE et al (2011) Mutations in 2 distinct genetic pathways result in cerebral cavernous malformations in mice. J Clin Invest 121, 1871-1881

38. Yoruk B, Gillers BS, Chi NC and Scott IC (2012) Ccm3 functions in a manner distinct from $\mathrm{Ccm} 1$ and $\mathrm{Ccm} 2$ in a zebrafish model of CCM vascular disease. Dev Biol 362, 121-131

39. Song $Y$, Eng $M$ and Ghabrial AS (2013) Focal defects in single-celled tubes mutant for Cerebral cavernous malformation 3, GCKIII, or NSF2. Dev Cell 25, 507-519

40. Zawistowski JS, Stalheim L, Uhlik MT et al (2005) CCM1 and CCM2 protein interactions in cell signaling: implications for cerebral cavernous malformations pathogenesis. Hum Mol Genet 14, 2521-2531

41. Fisher OS, Liu W, Zhang R et al (2015) Structural basis for 
the disruption of the cerebral cavernous malformations 2 (CCM2) interaction with Krev interaction trapped 1 (KRIT1) by disease-associated mutations. J Biol Chem 290, 2842-2853

42. Zhu Y, Wu Q, Xu JF et al (2010) Differential angiogenesis function of $\mathrm{CCM} 2$ and $\mathrm{CCM} 3$ in cerebral cavernous malformations. Neurosurg Focus 29, E1

43. McDonald DA, Shi C, Shenkar R et al (2014) Lesions from patients with sporadic cerebral cavernous malformations harbor somatic mutations in the CCM genes: evidence for a common biochemical pathway for CCM pathogenesis. Hum Mol Genet 23, 4357-4370

44. Gault J, Shenkar R, Recksiek P and Awad IA (2005) Biallelic somatic and germ line CCM1 truncating mutations in a cerebral cavernous malformation lesion. Stroke 36, 872-874

45. Kehrer-Sawatzki H, Wilda M, Braun VM, Richter HP and Hameister H (2002) Mutation and expression analysis of the KRIT1 gene associated with cerebral cavernous malformations (CCM1). Acta Neuropathol 104, 231-240

46. Pagenstecher A, Stahl S, Sure U and Felbor U (2009) A two-hit mechanism causes cerebral cavernous malformations: complete inactivation of CCM1, CCM2 or CCM3 in affected endothelial cells. Hum Mol Genet 18, 911-918

47. Jaffe $A B$ and Hall $A$ (2005) Rho GTPases: biochemistry and biology. Annu Rev Cell Dev Biol 21, 247-269

48. Borikova AL, Dibble CF, Sciaky N et al (2010) Rho kinase inhibition rescues the endothelial cell cerebral cavernous malformation phenotype. J Biol Chem 285, 11760-11764

49. Faurobert E, Rome C, Lisowska J et al (2013) CCM1-ICAP-1 complex controls beta1 integrin-dependent endothelial contractility and fibronectin remodeling. J Cell Biol 202, 545-561

50. Whitehead KJ, Chan AC, Navankasattusas S et al (2009) The cerebral cavernous malformation signaling pathway promotes vascular integrity via Rho GTPases. Nat Med 15, 177-184

51. Wustehube J, Bartol A, Liebler SS et al (2010) Cerebral cavernous malformation protein CCM1 inhibits sprouting angiogenesis by activating DELTA-NOTCH signaling. Proc Natl Acad Sci U S A 107, 12640-12645

52. Brutsch R, Liebler SS, Wustehube J et al (2010) Integrin cytoplasmic domain-associated protein-1 attenuates sprouting angiogenesis. Circ Res 107, 592-601

53. You C, Sandalcioglu IE, Dammann P, Felbor U, Sure U and Zhu Y (2013) Loss of CCM3 impairs DLL4-Notch signalling: implication in endothelial angiogenesis and in inherited cerebral cavernous malformations. J Cell Mol Med 17, 407-418

54. Marchi S, Corricelli M, Trapani E et al (2015) Defective autophagy is a key feature of cerebral cavernous malformations. EMBO Mol Med 7, 1403-1417

55. DiStefano PV, Kuebel JM, Sarelius IH and Glading AJ (2014) KRIT1 protein depletion modifies endothelial cell behavior via increased vascular endothelial growth factor (VEGF) signaling. J Biol Chem 289, 33054-33065

56. Zhu Y, Zhao K, Prinz A et al (2015) Loss of endothelial programmed cell death 10 activates glioblastoma cells and promotes tumor growth. Neuro Oncol 18, 538-548

57. Gore AV, Lampugnani MG, Dye L, Dejana $\mathrm{E}$ and
Weinstein BM (2008) Combinatorial interaction between CCM pathway genes precipitates hemorrhagic stroke. Dis Model Mech 1, 275-281

58. Rinaldi C, Bramanti P, Fama A et al (2015) Glyoxalase I A111e, Paraoxonase 1 Q192r and L55m Polymorphisms in Italian Patients with Sporadic Cerebral Cavernous Malformations: A Pilot Study. J Biol Regul Homeost Agents 29, 493-500

59. Guazzi P, Goitre L, Ferro E et al (2012) Identification of the Kelch Family Protein Nd1-L as a Novel Molecular Interactor of KRIT1. PLoS One 7, e44705

60. Boulday G, Rudini N, Maddaluno L et al (2011) Developmental timing of CCM2 loss influences cerebral cavernous malformations in mice. J Exp Med 208, 1835-1847

61. McDonald DA, Shenkar R, Shi C et al (2011) A novel mouse model of cerebral cavernous malformations based on the two-hit mutation hypothesis recapitulates the human disease. Hum Mol Genet 20, 211-222

62. Rosen JN, Sogah VM, Ye LY and Mably JD (2013) ccm2-like is required for cardiovascular development as a novel component of the Heg-CCM pathway. Dev Biol 376, 74-85

63. Zhou Z, Rawnsley DR, Goddard LM et al (2015) The cerebral cavernous malformation pathway controls cardiac development via regulation of endocardial MEKK3 signaling and KLF expression. Dev Cell 32, 168-180

64. Cullere X, Plovie E, Bennett PM, MacRae CA and Mayadas TN (2015) The cerebral cavernous malformation proteins $\mathrm{CCM} 2 \mathrm{~L}$ and $\mathrm{CCM} 2$ prevent the activation of the MAP kinase MEKK3. Proc Natl Acad Sci U S A 112, 14284-14289

65. Zheng X, Riant F, Bergametti F et al (2014) Cerebral cavernous malformations arise independent of the heart of glass receptor. Stroke 45, 1505-1509

66. Plummer NW, Gallione CJ, Srinivasan S, Zawistowski JS, Louis DN and Marchuk DA (2004) Loss of p53 sensitizes mice with a mutation in Ccm1 (KRIT1) to development of cerebral vascular malformations. Am J Pathol 165, 1509-1518

67. Shenkar R, Shi C, Rebeiz T et al (2015) Exceptional aggressiveness of cerebral cavernous malformation disease associated with PDCD10 mutations. Genet Med 17, 188-196

68. Stahl S, Gaetzner S, Voss K et al (2008) Novel CCM1, CCM2, and CCM3 mutations in patients with cerebral cavernous malformations: in-frame deletion in CCM2 prevents formation of a CCM1/CCM2/CCM3 protein complex. Hum Mutat 29, 709-717

69. Voss K, Stahl S, Schleider E et al (2007) CCM3 interacts with CCM2 indicating common pathogenesis for cerebral cavernous malformations. Neurogenetics 8, 249-256

70. Goudreault M, D'Ambrosio LM, Kean MJ et al (2009) A PP2A phosphatase high density interaction network identifies a novel striatin-interacting phosphatase and kinase complex linked to the cerebral cavernous malformation 3 (CCM3) protein. Mol Cell Proteomics 8, 157-171

71. Edelmann AR, Schwartz-Baxter S, Dibble CF et al (2014) Systems biology and proteomic analysis of cerebral cavernous malformation. Expert Rev Proteomics 11, 395-404

72. Hilder TL, Malone MH, Bencharit S et al (2007) Proteomic 
identification of the cerebral cavernous malformation signaling complex. J Proteome Res 6, 4343-4355

73. Draheim KM, Li X, Zhang R et al (2015) CCM2-CCM3 interaction stabilizes their protein expression and permits endothelial network formation. J Cell Biol 208, 987-1001

74. Li X, Zhang R, Zhang $\mathrm{H}$ et al (2010) Crystal structure of CCM3, a cerebral cavernous malformation protein critical for vascular integrity. J Biol Chem 285, 24099-24107

75. Fisher OS and Boggon TJ (2014) Signaling pathways and the cerebral cavernous malformations proteins: lessons from structural biology. Cell Mol Life Sci 71, 1881-1892

76. Liu W, Draheim KM, Zhang R, Calderwood DA and Boggon TJ (2013) Mechanism for KRIT1 release of ICAP1-mediated suppression of integrin activation. Mol Cell 49, 719-729

77. Gunel M, Laurans MS, Shin D et al (2002) KRIT1, a gene mutated in cerebral cavernous malformation, encodes a microtubule-associated protein. Proc Natl Acad Sci U S A 99, 10677-10682

78. Beraud-Dufour S, Gautier R, Albiges-Rizo C, Chardin P and Faurobert E (2007) Krit 1 interactions with microtubules and membranes are regulated by Rap1 and integrin cytoplasmic domain associated protein-1. FEBS J 274, 5518-5532

79. Liu JJ, Stockton RA, Gingras AR et al (2011) A mechanism of Rap1-induced stabilization of endothelial cell--cell junctions. Mol Biol Cell 22, 2509-2519

80. Li X, Zhang R, Draheim KM, Liu W, Calderwood DA and Boggon TJ (2012) Structural basis for small G protein effector interaction of Ras-related protein 1 (Rap1) and adaptor protein Krev interaction trapped 1 (KRIT1). J Biol Chem 287, 22317-22327

81. Gingras AR, Puzon-McLaughlin $\mathrm{W}$ and Ginsberg $\mathrm{MH}$ (2013) The structure of the ternary complex of Krev interaction trapped 1 (KRIT1) bound to both the Rap1 GTPase and the heart of glass (HEG1) cytoplasmic tail. J Biol Chem 288, 23639-23649

82. Stiegler AL, Zhang R, Liu W and Boggon TJ (2014) Structural determinants for binding of sorting nexin 17 (SNX17) to the cytoplasmic adaptor protein Krev interaction trapped 1 (KRIT1). J Biol Chem 289, 25362-25373

83. Fisher OS, Deng H, Liu D et al (2015) Structure and vascular function of MEKK3-cerebral cavernous malformations 2 complex. Nat Commun 6, 7937

84. Crose LE, Hilder TL, Sciaky N and Johnson GL (2009) Cerebral cavernous malformation 2 protein promotes smad ubiquitin regulatory factor 1-mediated RhoA degradation in endothelial cells. J Biol Chem 284, 13301-13305

85. Li X, Ji W, Zhang R, Folta-Stogniew E, Min W and Boggon TJ (2011) Molecular recognition of leucine-aspartate repeat (LD) motifs by the focal adhesion targeting homology domain of cerebral cavernous malformation 3 (CCM3). J Biol Chem 286, 26138-26147

86. Zhang M, Dong L, Shi Z et al (2013) Structural mechanism of CCM3 heterodimerization with GCKIII kinases. Structure 21, 680-688

87. Voss K, Stahl S, Hogan BM et al (2009) Functional analyses of human and zebrafish 18-amino acid in-frame deletion pave the way for domain mapping of the cerebral cavernous malformation 3 protein. Hum Mutat 30, 1003-1011

88. Ceccarelli DF, Laister RC, Mulligan VK et al (2011) CCM3/PDCD10 heterodimerizes with germinal center kinase III (GCKIII) proteins using a mechanism analogous to CCM3 homodimerization. J Biol Chem 286, 25056-25064

89. Fidalgo $M$, Fraile $M$, Pires $A$, Force $T$, Pombo $C$ and Zalvide J (2010) CCM3/PDCD10 stabilizes GCKIII proteins to promote Golgi assembly and cell orientation. J Cell Sci 123, 1274-1284

90. Kean MJ, Ceccarelli DF, Goudreault M et al (2011) Structure-function analysis of core STRIPAK Proteins: a signaling complex implicated in Golgi polarization. J Biol Chem 286, 25065-25075

91. He Y, Zhang H, Yu L et al (2010) Stabilization of VEGFR2 signaling by cerebral cavernous malformation 3 is critical for vascular development. Sci Signal 3, ra26

92. Dibble CF, Horst JA, Malone MH et al (2010) Defining the functional domain of programmed cell death 10 through its interactions with phosphatidylinositol-3,4,5-trisphosphate. PLoS One 5, e11740

93. Mouchtouris N, Chalouhi N, Chitale A et al (2015) Management of cerebral cavernous malformations: from diagnosis to treatment. ScientificWorldJournal 2015, 808314

94. McDonald DA, Shi C, Shenkar R et al (2012) Fasudil decreases lesion burden in a murine model of cerebral cavernous malformation disease. Stroke 43, 571-574

95. Westover MB, Bianchi MT, Eckman MH and Greenberg SM (2011) Statin use following intracerebral hemorrhage: a decision analysis. Arch Neurol 68, 573-579

96. Mikati AG, Khanna O, Zhang L et al (2015) Vascular permeability in cerebral cavernous malformations. J Cereb Blood Flow Metab 35, 1632-1639 